\title{
Optimal Observer Trajectory for Towed Sonar in Bearings-Only Tracking
}

\author{
Yingchun Chen, Xianhua Wang
}

Science \& Technology on Underwater Acoustic Antagonizing Laboratory, Zhanjiang 524022, P. R. China

cyc03@163.com

Keywords: Bearings-only tracking, towed sonar, optimal trajectory, genetic algorithm, least square method

\begin{abstract}
The unique constraints for the mother ship of towed sonar in bearings-only tracking is that it cann't maneuver frequently because during maneuvering and a certain time after maneuvering, the bearing measurements are not available. The maneuvering trajectories of the mother ship will greatly affect the locating and tracking accuracy. The least square method for estimating the motion parameters of the target is described. By considering these unique constraints, a genetic algorithm based on least squre method is adopted to optimize the observer trajectory. Simulation result shows the validity of the method.
\end{abstract}

\section{Introduction}

Compared with ship hull sonar, the towed sonar enjoys the characteristics of big aperture, low background noise and variable working depth, which resulting in its long-distance detection capability. But it also has its own limitations [1]. On one hand, it cannot range the target directly, which requires proper maneuvering for a relative long time. On the other hand, the flexible arrays limit the maneuvering of the mother ship. Researches [2] showed that when the towed sonar was used for Bearings-Only Tracking (BOT), its locating and tracking precision were greatly affected by the maneuvering trajectory of the mother ship.

In 1989, in order to locate a fixed target in a two-dimensional plane, S. E. Hammel and P. T. Liu [3] made fundamental researches on optimal observer motion for localization with bearings measurements. The performance index for optimization was maximizing the area of location error ellipse, or the determinant of Fisher Information Matrix. Based on this, J. P. Helferty, etc. [4] pointed out that there existed great uncertainty in choosing the maximization of area of location error eclipse as the performance index. In addition, it is very complex to computate the determinant of Fisher Information Matrix. In a replacement, minimizing the trace of Cramer-Rao Lower Bound was proposed as the performance index.

In 1998, J. M. Passerieux and D. V. Cappel [5] studied the optimal observer maneuvering for bearings-only target tracking on two-dimensional plane. The optimal global precision and distance precision were adopted as performance indexes. Then optimal control theory was used to construct a Hamilton function. The optimal maneuvering trajectories of the observer were composed by two or three segments of almost the same length. In 1999, O. Tremois and L. J. Cadre [6] studied the optimal trajectories of observers. They concluded that optimizing the determinant of FIM was almost equivalent to maximizing the rate between bearing variation ratio to the square of relative distance and that the optimal speed of observer should be the maximum speed of the observer.

The above researches took the Cramer-Rao lower bound of location precision, FIM matrix or their variances as optimization index. In order to compute the trace of Cramer-Rao lower bound or the determinant of FIM, the motion information of the target should be known. But that is not available. That is the ultimate goal of bearing only tracking. Therefore, the optimization of observer and the goal of BOT locating and tracking is a paradox.

As flexible arrays are used for towed sonar, only a certain stable time after maneuvering will the bearing measurements of the towed sonar be valid. Therefore, it is improper for the observer to maneuver frequently. That is the unique constraint in optimizing observer trajectory for the towed sonar in bearings-only tracking. 


\section{Model Description}

The geometrical scenario between the target and mother ship of towed sonar is shown in Fig. 1.

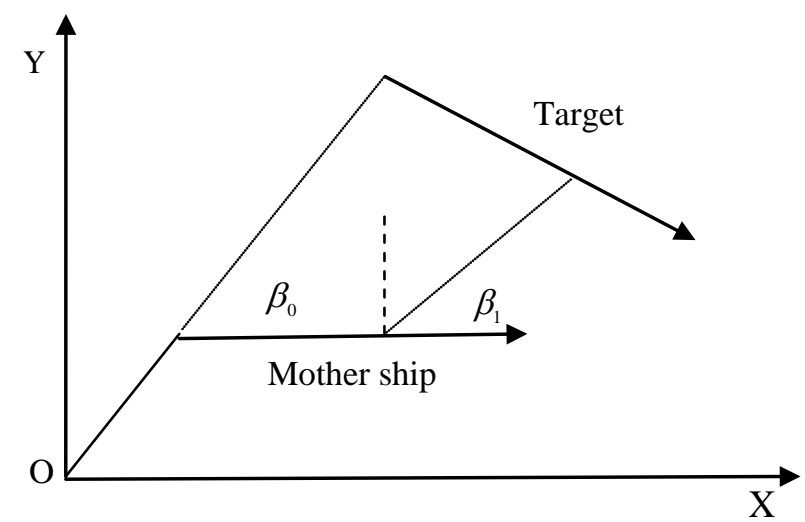

Fig. 1 Geometrical scenario between the target and mother ship

Take the position of mother ship of the towed sonar as the origin of rectangular coordinates system when it has just detected a target. Assume the target moves forward along course $c_{s}$ at a uniform speed $v_{s}$. Define decision variable $k_{D}$. If $k<k_{D}$, then the mother ship moves forward along course $c_{m}$ at uniform speed $v_{m}$; if $k=k_{D}$, the mother ship begins turning to the new course $c_{n}$ with maximum turn rate; if $k>\left\lfloor k_{D}+\left|c_{n}-c_{m}\right| R / v_{m}\right\rfloor$, the mother ship has finished turning and moves straightford along the new course $c_{n}$ at uniform speed $v_{m}$. Thus, under the rectangular coordinates system, the movement status of the target at time $k$ is determined by $X_{s}(k)=\left\lfloor\begin{array}{lllll}X_{s}(k) & y_{s}(k) & v_{s x} & v_{s y}\end{array}\right\rfloor$ while that of the mother ship is determined by $X_{m}(k)=\left\lfloor\begin{array}{llll}x_{m}(k) & y_{m}(k) & v_{m x}(k) & v_{m y}(k)\end{array}\right]$,

Where

$$
\begin{aligned}
& V_{s X}=v_{s} \sin c_{s}, V_{s y}=v_{s} \cos c_{s}, \\
& V_{m x}=v_{m} \sin c_{m}, V_{m y}=v_{m} \cos c_{m}, \\
& x_{s x}(k)=\int_{0}^{k \cdot \Delta T} v_{s x}(\tau) \mathrm{d} \tau, x_{s y}(k)=\int_{0}^{k \cdot \Delta T} v_{s y}(\tau) \mathrm{d} \tau, \\
& x_{m x}(k)=\int_{0}^{k \cdot \Delta T} v_{m x}(\tau) \mathrm{d} \tau, x_{m y}(k)=\int_{0}^{k \cdot \Delta T} v_{m y}(\tau) \mathrm{d} \tau .
\end{aligned}
$$

Thus, the relative movement state between the target and mother ship at time $k$ is notated as $X(k)=X_{s}(k)-X_{m}(k)$. The discrete state equation can be rewritten as [2]

$$
X(k)=\Phi(\Delta T) X(k-1)+U(k)
$$

Where $\Delta T$ stands for the sampling period of system;

$\Phi(\Delta T)=\left[\begin{array}{cccc}1 & 0 & \Delta T & 0 \\ 0 & 1 & 0 & \Delta T \\ 0 & 0 & 1 & 0 \\ 0 & 0 & 0 & 1\end{array}\right]$ stands for the state transform matrix;

$U(k)=\left\lfloor\begin{array}{llll}0 & 0 & u_{m x}(k) & u_{m y}(k)\end{array}\right\rfloor$ stands for the maneuvering of the mother ship of the towed sonar.

The observer equation of system can be written as follows [2], 


$$
\beta_{k}=\arctan \left(\left(x_{s}(k)-x_{m}(k)\right) /\left(y_{s}(k)-y_{m}(k)\right)\right)+e_{k}
$$

Where $e_{k}$ stands for stachastic observing noise with mean value zero and variance $\sigma_{k}^{2}$.

As assuming the target moves forward at uniform speed, the position of the target at any time $k$ can be calculated from its initial state vector according to Eq. 4,

$$
\begin{aligned}
& x_{s}(k)=x_{s}(0)+k \cdot \Delta T \cdot v_{s x}, \\
& y_{s}(k)=y_{s}(0)+k \cdot \Delta T \cdot v_{s y} .
\end{aligned}
$$

Therefore, Eq. 3 can be rewritten as

$$
\beta_{k}=\arctan \left(\left(x_{s}(0)+k \cdot \Delta T \cdot v_{s_{X}}-x_{m}(k)\right) /\left(y_{s}(0)+k \cdot \Delta T \cdot v_{s y}-y_{m}(k)\right)\right)+e_{k} \cdot
$$

Expand Eq. 5 and obtain [2]

$$
x_{m}(k) \cos \beta_{k}-y_{m}(k) \sin \beta_{k}=x_{s}(0) \cos \beta_{i}-y_{T}(0) \sin \beta_{i}+i \cdot \Delta T \cos \beta_{i}-i \cdot \Delta T \sin \beta_{i}+\varepsilon_{i},
$$

Where

$\varepsilon_{i}=r_{i} \tan e_{i}$,

$r_{i}=\left(x_{T}(0)+i \Delta T v_{T x}-x_{0}(i)\right) \sin \beta_{i}+\left(y_{T}(0)+i \cdot \Delta T \cdot v_{T y}-y_{0}(i)\right) \cos \beta_{i}$,

$i=1,2,3, \cdots$.

After having observed $K$ bearing measurements and written as vectors, a new measurement equation is obtained [2]

$$
Z_{k}=A_{K}(\beta) \cdot X_{T}(0)+\varepsilon_{K},
$$

Where

$$
\begin{aligned}
& Z_{K}=\left(x_{0}(i) \cos \beta_{i}-y_{0}(i) \sin \beta_{i} \quad \cdots \quad x_{0}(i) \cos \beta_{i}-y_{0}(i) \sin \beta_{i}\right)^{T} \\
& =\left(\begin{array}{lll}
z_{o} & \cdots & z_{k}
\end{array}\right)^{T} \\
& A_{K}(\beta)=\left(\begin{array}{cccc}
\cos \beta_{0} & -\sin \beta_{0} & 0 \cdot \Delta T \cdot \cos \beta_{0} & -0 \cdot \Delta T \cdot \sin \beta_{0} \\
\vdots & \vdots & \vdots & \vdots \\
\cos \beta_{k} & -\sin \beta_{k} & k \cdot \Delta T \cdot \cos \beta_{k} & -k T \sin \beta_{0}
\end{array}\right)=\left(\begin{array}{l}
a_{0} \\
\vdots \\
a_{k}
\end{array}\right), \\
& \varepsilon_{K}=\left(\varepsilon_{0} \cdots \varepsilon_{k}\right)^{T}, k=0,1,2, \cdots, K \text {. }
\end{aligned}
$$

Applying least square method to Eq. 7, the estimation of least square method for the initial state of the target can be obtained

$$
A_{k}^{T}(\beta) \cdot A_{k}(\beta) \cdot X_{t}(0)=A_{k}^{T}(\beta) \cdot Z_{k}
$$

Define matrixes as follows, 


$$
\begin{aligned}
& G=A_{K}^{T}(\beta) \cdot A_{K}(\beta) \\
& =\sum_{i=0}^{K}\left[\begin{array}{cccc}
\cos ^{2} \beta_{i} & -\sin \beta_{i} \cos \beta_{i} & i \cdot \Delta T \cdot \cos ^{2} \beta_{i} & -i \cdot \Delta T \cdot \sin \beta_{i} \cos \beta_{i} \\
-\sin \beta_{i} \cos \beta_{i} & \sin ^{2} \beta_{i} & -i \cdot \Delta T \cdot \sin \beta_{i} \cos \beta_{i} & i \cdot \Delta T \cdot \sin ^{2} \beta_{i} \\
i \cdot \Delta T \cdot \cos ^{2} \beta_{i} & -i \cdot \Delta T \cdot \sin \beta_{i} \cos \beta_{i} & (i \cdot \Delta T)^{2} \cos ^{2} \beta_{i} & -(i \cdot \Delta T)^{2} \sin \beta_{i} \cos \beta_{i} \\
-i \cdot \Delta T \cdot \sin \beta_{i} \cos \beta_{k} & i \cdot \Delta T \cdot \sin ^{2} \beta_{k} & -(i \cdot \Delta T)^{2} \sin \beta_{i} \cos \beta_{i} & (i \cdot \Delta T)^{2} \sin ^{2} \beta_{i}
\end{array}\right], \\
& B=A_{K}^{T}(\beta) Z \\
& =\sum_{i=0}^{K}\left[\begin{array}{l}
x_{0}(i) \cos ^{2} \beta_{i}-y_{0}(i) \sin \beta_{i} \cos \beta_{i} \\
-x_{0}(i) \sin \beta_{i} \cos \beta_{i}+y_{0}(i) \sin ^{2} \beta_{i} \\
i \cdot \Delta T \cdot x_{0}(i) \cos ^{2} \beta_{i}-i \cdot \Delta T \cdot y_{0}(i) \sin \beta_{i} \cos \beta_{i} \\
-i \cdot \Delta T \cdot x_{0}(i) \sin \beta_{i} \cos \beta_{i}+i \cdot \Delta T \cdot y_{0}(i) \sin ^{2} \beta_{i}
\end{array}\right] .
\end{aligned}
$$

If $G$ is reversible, Eq. 7 has unique solution

$$
X_{t}(0)=G^{-1} \cdot B
$$

Based on Eq. 11, the initial state of the target can be obtained.

\section{Algorithm Description}

Virtue of Genetic Algorithm. Genetic Algorithm (GA) has been applied to find optimal solutions to various combinatorial problems since its emergence. Compared with conventional methods, this innovative solution technique works in a different way. Many researchers used GA to solve complex real-world problems and obtained good results from these techniques. There are two distinguishing characteristics of GA that separate GA from the general optimization techniques. The first is that GA starts with an initial set of random feasible solutions, instead of a single solution. GA generates many feasible solutions to a given problem and let them compete and mate so as to generate improved offsprings. The second characteristic is that GA does not have any specific functional requirement for the mathematical relationships that express a given problem. Therefore GA can handle with any kind of objective functions and constraints.

Design of Genetic Operators. The chromosome is divided into several gene segments to represent the initial course of observer $c_{m}$ (rad.), the maneuvering time of observer $k_{D}(\mathrm{~s})$, and the new course after maneuvering $c_{n}$ (rad.). Floating point numbers in $[0,1]$ are used to represent genes, which greatly simplifies the crossover, mutation and other operations. As the ranges of gene values are not the same, the following proportional and translation transformations are used for each gene before fitness evaluation,

$$
\begin{aligned}
& k_{D}=10+1000 f_{1}, \\
& c_{m}=\left(f_{2}-0.5\right) 2 \pi, \\
& c_{n}=\left(f_{3}-0.5\right) 2 \pi .
\end{aligned}
$$

Where, $f_{i}, i=1,2,3$ are floating numbers in interval $[0,1]$, generated by GA.

Then the turning time of the mother ship can be calculated as follows,

$$
t_{\text {turn }}=\left(c_{n}-c_{m}\right) / \arcsin \left(v_{m} / R_{\text {turn }}\right) \text {, }
$$

Where $R_{\text {turn }}$ is the maximum turning radius of the mother ship. 
Fitness of GA. The objective for the towed sonar is to track target precisely. Thus, the fitness function is defined as

$$
\text { fitness }= \begin{cases}1, & \text { BOT converges and } \mathrm{e}<1, \\ 1 / e, & \text { BOT converges and } \mathrm{e}>1, \\ 0, & \text { BOT doesn't converge. }\end{cases}
$$

Where

$$
e=\frac{1}{K}\left(\sum_{i=1}^{k_{D}+t_{\text {turn }}} \sqrt{\left(x_{s}(i)-x_{s}^{\prime}(i)\right)^{2}+\left(y_{s}(i)-y_{s}^{\prime}(i)\right)^{2}}+\sum_{i=k_{D}+t_{\text {turn }}+1}^{K} \sqrt{\left.\left(x_{s}(i)-x_{s}^{\prime}(i)\right)^{2}+\left(y_{s}(i)-y_{s}^{\prime}(i)\right)^{2}\right)}\right.
$$

represents the estimated distance error,

$\left(x_{s}(i), y_{s}(i)\right)$ represents the real positions of target at time $i$,

$\left(x_{s}^{\prime}(i), y_{s}^{\prime}(i)\right)$ represents the estimated positions of the target at time $i$.

Process of GA. Ratio selection operator, elite keeping strategy, and two-point mutation are adopted in GA. The optimization process of GA is described as follows.

Step 1: The evolutionary generation and setup parameters are initialized and the initial population is generated randomly.

Step 2: Once the setup parameters and genes of a chromosome are given, the mother ship, and the target move according to their trajectory models respectively. When the bearings-only tracking algorithm based on least-square-method converges, Eq. 14 is used to calculate fitness.

Step 3: Evolutionary process:

a) Crossover operation of individuals.

b) Mutation operation of individuals.

c) The fitness of the population is evaluated based on the outcome of bearings-only tracking.

d) Selection and copy operation of individual.

Step 4: Increase the number of generation. If it reaches the maximum evolutionary generation, the algorithm terminates after outputting the optimal individual; otherwise goes back to Step 3 for further evolution.

\section{Simulation Result}

Initial Conditions. The initial conditions of simulation are set up as follows [5].

The observer is located at $(0,0)$ at the very beginning and the speed of the observer is $3 \mathrm{~m} / \mathrm{s}$. The target is located at $(0,10 \mathrm{~km})$ at the very beginning and moves with straight line along the direction of $330^{\circ}$ (based on the rectangular system) at a speed of $5 \mathrm{~m} / \mathrm{s}$. The turn radius of the observer is $30 \mathrm{~m}$. After the observer has turned to a new direction, the towed sonar waits 120 seconds for its arrays to be ready. I.e., during the turning period and the very first 120 seconds at the new direction, no measurement data will be available for the towed sonar. For the other time, the towed sonar measures the bearing every second. The measurement error is root mean square $0.5^{\circ}$.

Uniform mutation, proportional selection with elite replacement strategy and single point crossover operators are adopted. The size of population is 100 and evolutionary generation is 100 . The crossover probability is 0.85 and mutation probability is 0.10 .

Simulation Result. According to the above-mentioned mathematical model and given parameters, the optimized parameters of observer trajectory are as follows.

The initial direction is $c_{m}=165.92^{\circ}$, and the observer moves along this direction for $k_{D}=374 \mathrm{~s}$. The the observer turns to the new direction of $c_{n}=35.15^{\circ}$. The estimated initial positions of the target is $(171.21 \mathrm{~m}, 9228.54 \mathrm{~m})$, and the estimated distance error is $138.12 \mathrm{~m}$. The target trajectory and optimal observer trajectory are shown in Fig. 2. 


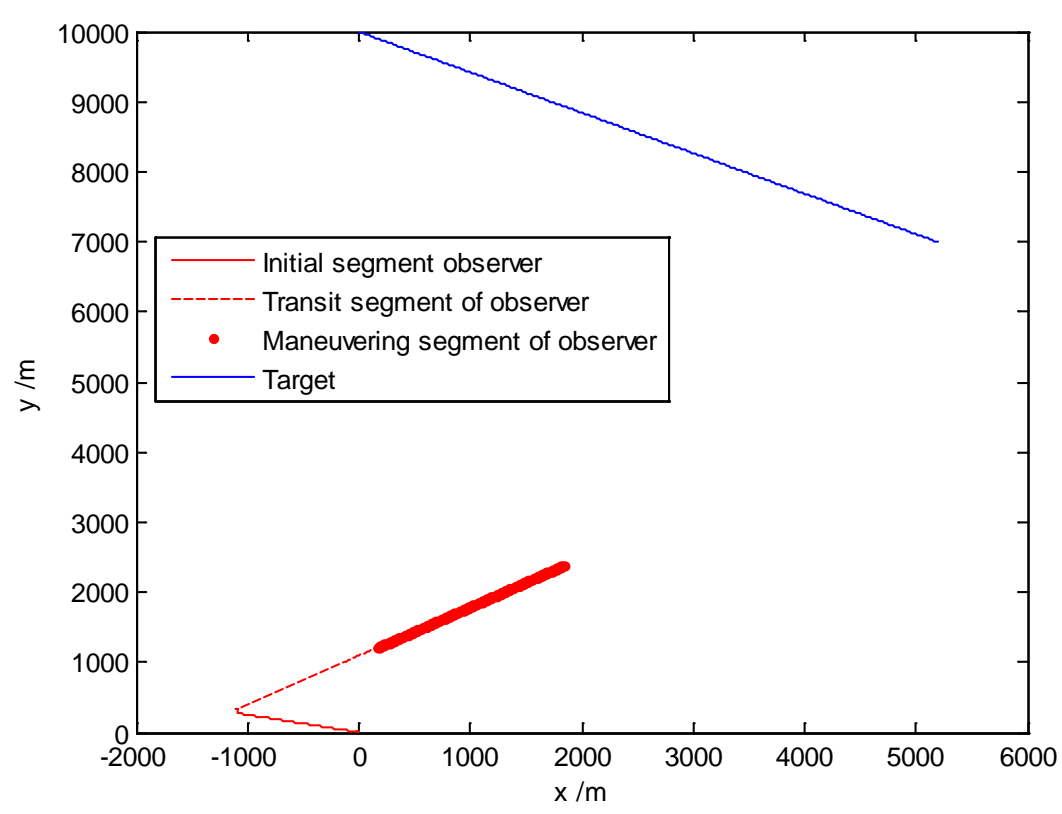

Fig. 2 Target trajectory and optimal observer trajectory

\section{Conclusions}

Considering the trajectory constraints unique to mother ship of the towed sonar in bearings-only tracking, a least-square-method based genetic algorithm is adopted to optimize the observer trajectory. Simulation result shows the validity of the method. In future work, the particle filter and extended Kalman filter will be used to replace the least square method in estimating the motion parameters of the target. In order to test the robustness of the observer trajectory, other forms of observer and target motion will also be taken into consideration.

\section{References}

[1] L. Yang, Z. L. Yuan, and D. D. Liu: Ship-helicopter coalition antisubmarine application of accounterment towed sonar, Ordance Industry Automation, vol. 28, no. 7, pp. 42-44 (2009).

[2] Z. S. Shi, Z. Liu, H. Y. Wang, and etc.: Method and Theory of Target Tracking and Data Fusion, National Defense Industry Press, P.R. China (2010).

[3] S. E. Hammel, and P. T. Liu: Optimal observer motion for localization with bearings measurements, Computers Maths. Application, vol. 18, no. 1, pp. 171-180 (1989).

[4] J. P. Helferty, and D. R. Mudgett: Optimal observer trajectories for bearings-only tracking by minimizing the trace of the Cramer-Rao lower bound, in: Proc. Of 32th International Conference of Control and Decision, pp. 936-938 (1993).

[5] J. M. Passerieux, and D. V. Cappel: Optimal observer maneuvering for bearings-only target tracking, IEEE Trans. On Aerospace and Electronic System, vol. 34, no. 3, pp. 777-788 (1998).

[6] O. Tremois, and L. J. Cadre: Optimal observer trajectory in bearings-only tracking for maneuvering sources, in: IEE Proc. Of Radar Sonar Navigation, vol. 146, no. 1, pp. 31-39 (1999). 